\title{
Anyons as quon particles
}

\author{
V.Bardek, M.Dorešić, and S.Meljanac" \\ Department of Theoretical Physics, \\ Rudjer Bošković Institute, P.O.B. 1016, \\ 41001 Zagreb,CROATIA
}

\begin{abstract}
The momentum operator representation of nonrelativistic anyons is developed in the Chern - Simons formulation of fractional statistics. The connection between anyons and the q-deformed bosonic algebra is established.
\end{abstract}

PACS numbers: 03.65.-w,03.70.+k,05.30.-d,11.10.Ln.

\footnotetext{
${ }^{1}$ e-mail address: bardek@thphys.irb.hr

e-mail address: doresic@thphys.irb.hr

e-mail address: meljanac@thphys.irb.hr
} 
It is a well-known phenomenon that particles in $2+1$ dimensions minimally coupled to an Abelian gauge field with the Chern - Simons Lagrangian change their spin and statistics [1].The corresponding vector potential can be absorbed in a new matter field that obeys graded, anyonic commutation relations [2]. Commutators are changed into anticommutators for special values of the Chern - Simons parameter.For other values ,the interpolating anyon statistics appears. This is the essence of the so-called statistical transmutation.

Floratos and Tomaras [0] have recently proposed a connection between the $\mathrm{q}$ - deformed harmonic oscillator and the discretized two-anyon systems, with q being a primitive root of unity and the relative radial motion of anyons frozen.

On the other hand,Greenberg [4] has shown that the q-deformation ( q being a real parameter )of the bilinear Bose (or Fermi) commutation algebra introduces particles called "quons" that interpolate between fermions and bosons.

In this paper we develop a momentum-operator representation of nonrelativistic anyons.We demonstrate that anyons can be alternatively represented as a q-deformation of an underlying bosonic algebra.This can be viewed as an extension of Greenberg's approach with q being a complex number, $|q|=1$. Furthermore,we construct $\mathcal{R}$-matrices for anyon operators both in position and momentum space and show that they satisfy the Yang-Baxter equation and the Hecke condition.

The quantum-mechanical problem of nonrelativistic bosons interacting with the U(1) gauge field described by the Chern-Simons Lagrangian has been formulated and solved by Jackiw and Pi [5]. They have shown that the corresponding Hamiltonian can be reduced to the following form:

$$
H=\frac{1}{2} \int d^{2} r \vec{\Pi}^{\dagger}(\vec{r}) \vec{\Pi}(\vec{r}) .
$$


The operator covariant derivative $\vec{\Pi}$ is given by

$$
\vec{\Pi}(\vec{r})=(\vec{\nabla}-i \vec{A}(\vec{r})) \Psi(\vec{r}) .
$$

The quantum-field operator $\Psi(\vec{r})$ and its Hermitian conjugate $\Psi^{\dagger}(\vec{r})$ obey the bosonic commutation relations at equal times:

$$
\begin{aligned}
{\left[\Psi(\vec{r}), \Psi\left(\vec{r}^{\prime}\right)\right] } & =0, \\
{\left[\Psi^{\dagger}(\vec{r}), \Psi^{\dagger}\left(\vec{r}^{\prime}\right)\right] } & =0, \\
{\left[\Psi(\vec{r}), \Psi^{\dagger}\left(\vec{r}^{\prime}\right)\right] } & =\delta\left(\vec{r}-\vec{r}^{\prime}\right) .
\end{aligned}
$$

The vector potential $\vec{A}$ can be completely described by the bosonic number density operator $\rho$ :

$$
\begin{gathered}
\vec{A}(\vec{r})=-\lambda \hat{n} \times \int \frac{\vec{r}-\vec{r}^{\prime}}{\left|\vec{r}-\vec{r}^{\prime}\right|^{2}} \rho\left(\vec{r}^{\prime}\right) d^{2} r^{\prime}, \\
\rho(\vec{r})=\Psi^{\dagger}(\vec{r}) \Psi(\vec{r}),
\end{gathered}
$$

where $\lambda$ is the so-called statistical parameter whose role will be clarified below.In other words, the gauge field has no independent dynamics.In this way,the vector potential is completely eliminated at the expense of the Hamiltonian becoming nonlocal.

As has been noted in [6], the definition of $\vec{A}$ as a pure gauge is

$$
\vec{A}(\vec{r})=\vec{\nabla} \omega(\vec{r}),
$$

where

$$
\omega(\vec{r})=-\lambda \int \theta\left(\vec{r}-\vec{r}^{\prime}\right) \rho\left(\vec{r}^{\prime}\right) d^{2} r^{\prime} .
$$

This definition enables one to transform the nonlocal interaction away.However, the polar angel $\theta$ has to be a multivalued function; otherwise there would be residual interactions originating from the cut.By the phase redefinition of the bosonic field,

$$
\Psi(\vec{r})=e^{i \omega(\vec{r})} \tilde{\Psi}(\vec{r}),
$$


we obtain the following free Hamiltonian of the new anyonic field $\tilde{\Psi}$ :

$$
\tilde{H}=\frac{1}{2} \int d^{2} r \vec{\nabla} \tilde{\Psi}^{\dagger}(\vec{r}) \vec{\nabla} \tilde{\Psi}(\vec{r})
$$

The commutation relations (3) are now modified as

$$
\begin{aligned}
\tilde{\Psi}(\vec{r}) \tilde{\Psi}\left(\vec{r}^{\prime}\right)-e^{-i \lambda \Delta\left(\vec{r}-\vec{r}^{\prime}\right)} \tilde{\Psi}\left(\vec{r}^{\prime}\right) \tilde{\Psi}(\vec{r}) & =0, \\
\tilde{\Psi}^{\dagger}(\vec{r}) \tilde{\Psi}^{\dagger}\left(\vec{r}^{\prime}\right)-e^{-i \lambda \Delta\left(\vec{r}-\vec{r}^{\prime}\right)} \tilde{\Psi}^{\dagger}\left(\vec{r}^{\prime}\right) \tilde{\Psi}^{\dagger}(\vec{r}) & =0, \\
\tilde{\Psi}(\vec{r}) \tilde{\Psi}^{\dagger}\left(\vec{r}^{\prime}\right)-e^{i \lambda \Delta\left(\vec{r}-\vec{r}^{\prime}\right)} \tilde{\Psi}^{\dagger}\left(\vec{r}^{\prime}\right) \tilde{\Psi}(\vec{r}) & =\delta\left(\vec{r}-\vec{r}^{\prime}\right) .
\end{aligned}
$$

Here $\Delta$ denotes the difference

$$
\Delta\left(\vec{r}-\vec{r}^{\prime}\right)=\theta\left(\vec{r}-\vec{r}^{\prime}\right)-\theta\left(\vec{r}^{\prime}-\vec{r}\right)
$$

Because of the multivaluedness of $\theta$, this difference can be identified with the infinite set $\{\pi+2 \pi z\}, \mathrm{z}$ being an integer. When $\lambda$ takes a rational value, the multivaluedness is finite. The multivalued nature of the phase factor $\Delta$ is essential to the consistency of the graded commutation relations (10). The excitations described by the new field $\tilde{\Psi}$ obey fractional statistics.

For $\lambda=0 \bmod 2$, we have bosons; for $\lambda=1 \bmod 2$, we have fermions, whereas for an arbitrary value of $\lambda$, we have anyons. The graded commutators (10) resemble the q-deformed algebras considered by Greenberg [4]. Note,however,that this algebra was formulated in momentum space. In order to compare and further analyze such algebras,one should be able to evaluate a full quantum-field theoretic description of anyons in momentum space.Hence,we should Fourier transform the anyonic fields and the graded commutation relations in Eq. (10).However,the problem is how to define the integration of a multivalued function precisely;for example, in Eq. (7).

We define the integration of a multivalued function as a set of integrals of single-valued functions defined in the correspoding sheets of the Riemann surface. This set of single-valued functions we then treat as one multivalued function. 
Now we apply this definition to the multivalued angle function $\theta(\vec{r})$.In the zeroth sheet, we define polar angle as $\theta_{0}(\vec{r})$ with $\theta_{0}(\vec{r}) \in[0,2 \pi)$ which satisfies

$$
\theta_{0}(\vec{r})-\theta_{0}(-\vec{r})=\Delta_{0}(\vec{r})
$$

where

$$
\Delta_{0}(\vec{r})= \begin{cases}-\pi \operatorname{sgn} y(\vec{r}) & \text { if } y \neq 0 \\ -\pi \operatorname{sgn} x(\vec{r}) & \text { if } y=0 .\end{cases}
$$

The multivalued angle function is

$$
\begin{aligned}
\theta(\vec{r}) & =\theta_{0}(\vec{r})+2 \pi z, \quad z \in Z, \\
\theta(-\vec{r}) & =\theta_{0}(-\vec{r})+\Delta_{0}(\vec{r})-\Delta,
\end{aligned}
$$

satisfying

$$
\theta(\vec{r})-\theta(-\vec{r})=\Delta=\pi+2 \pi z, z \in Z
$$

The Fourier transform of the multivalued function is calculated according to (using) our definition of the integration of the multivalued function.For example, let us consider multivalued constant $\Delta \equiv \pi+2 \pi z, z \in Z$. In the $\mathrm{n}$-th sheet, the value is $\pi+2 \pi n$ and its Fourier transform is $(\pi+2 \pi n) \delta(\vec{k})$. Hence the Fourier transform of the multivalued constant in momentum space is $(\pi+2 \pi z) \delta(\vec{k})$.

Let us now construct annihilation and creation anyon operators in momentum space by Fourier transforming the anyon field $\tilde{\Psi}$ and its Hermitian conjugate $\tilde{\Psi}^{\dagger}$ :

$$
\begin{aligned}
\tilde{a}(\vec{k}) & =\frac{1}{2 \pi} \int e^{-i \vec{k} \vec{r}} \tilde{\Psi}(\vec{r}) d^{2} r \\
\tilde{a}^{\dagger}(\vec{k}) & =\frac{1}{2 \pi} \int e^{i \vec{k} \vec{r}} \tilde{\Psi}^{\dagger}(\vec{r}) d^{2} r
\end{aligned}
$$

In terms of the above operators, the commutator algebra (10) translates into

$$
\tilde{a}(\vec{p}) \tilde{a}(\vec{q})-\frac{1}{4 \pi^{2}} \int d^{2} k \int d^{2} r e^{-i \lambda \Delta(\vec{r})+i \vec{k} \vec{r}} \tilde{a}(\vec{q}-\vec{k}) \tilde{a}(\vec{p}+\vec{k})=0,
$$




$$
\begin{aligned}
\tilde{a}^{\dagger}(\vec{p}) \tilde{a}^{\dagger}(\vec{q})-\frac{1}{4 \pi^{2}} \int d^{2} k \int d^{2} r e^{-i \lambda \Delta(\vec{r})+i \vec{k} \vec{r}} \tilde{a}^{\dagger}(\vec{q}+\vec{k}) \tilde{a}^{\dagger}(\vec{p}-\vec{k}) & =0, \\
\tilde{a}(\vec{p}) \tilde{a}^{\dagger}(\vec{q})-\frac{1}{4 \pi^{2}} \int d^{2} k \int d^{2} r e^{i \lambda \Delta(\vec{r})+i \vec{k} \vec{r}} \tilde{a}^{\dagger}(\vec{q}+\vec{k}) \tilde{a}(\vec{p}+\vec{k}) & =\delta(\vec{p}-\vec{q}) .
\end{aligned}
$$

For anyonic values of the statistical parameter $\lambda$, the graded commutation relations (17) imply the hard-core condition on one-particle operators:

$$
\tilde{a}^{2}(\vec{p})=0 \quad, \quad \tilde{a}^{\dagger}(\vec{p})=0
$$

From relation (11) we know that $\Delta$ is actually a multivalued constant and can therefore be removed outside the integration in (17). The resulting algebra has the same form as the algebra in position space (10). It is obvious that the connection with a q-deformed algebra is a direct one.We simply identify the qparameter and the multivalued unimodular complex number $e^{i \lambda \pi(1+2 z)}$. This is,in some sense, a generalization of the usual (standard) notion of q-deformed field theory considered by Greenberg.By substituting the Fourier decomposition of the anyon fields $\tilde{\Psi}$ (16) into the free Hamiltonian (9) , we can easily obtain the corresponding Hamiltonian in momentum space:

$$
\tilde{H}=\frac{1}{2} \int d^{2} p \vec{p}^{2} \tilde{a}^{\dagger}(\vec{p}) \tilde{a}(\vec{p})
$$

where $\vec{p}^{2} / 2$ is the single-particle energy for a noninteracting system. The Hamiltonian operates in the Fock space generated by repeated application of the creation operators $\tilde{a}^{\dagger}(\vec{p})$ to the vacuum $\mid 0>$, which is, by definition, annihilated by all the operators $\tilde{a}(\vec{p})$ :

$$
\tilde{a}(\vec{p}) \mid 0>=0 \text { for all } \vec{p}
$$

Using the commutation algebra (17) and the hard-core condition (18), it is easily verified that all states in our momentum space have a non-negative squared norm. One can use the bosonic $(\mathrm{q}=1)$ operators $a(\vec{k})$ as building blocks to construct a 
momentum representation of the anyon operator in the general case $\left(q=e^{i \lambda \pi(1+2 z)} \neq 1\right)$.By Fourier transforming relation (8), we obtain

$$
\begin{aligned}
\tilde{a}(\vec{k})= & a(\vec{k})+i \lambda \int d^{2} k^{\prime} \theta\left(\overrightarrow{k^{\prime}}\right) \rho\left(\overrightarrow{k^{\prime}}\right) a\left(\vec{k}-\overrightarrow{k^{\prime}}\right) \\
& -\frac{\lambda^{2}}{2} \int d^{2} k^{\prime} d^{2} k^{\prime \prime} \theta\left(\overrightarrow{k^{\prime}}\right) \theta\left(\overrightarrow{k^{\prime \prime}}\right) \rho\left(\overrightarrow{k^{\prime}}\right) \rho\left(\overrightarrow{k^{\prime \prime}}\right) a\left(\vec{k}-\overrightarrow{k^{\prime}}-\overrightarrow{k^{\prime \prime}}\right)+\ldots,
\end{aligned}
$$

where we have formally expanded the exponential kernel in powers of the statistical parameter $\lambda . \rho(\vec{k})$ denotes the Fourier transform of the bosonic density $\rho(\vec{r})$, and $\theta(\vec{k})$ is given by

$$
\begin{aligned}
\theta(\vec{k}) & =\int d^{2} r e^{-i \vec{k} \vec{r}} \theta(\vec{r}) \\
& =4 \pi^{3}(1+2 z) \delta(\vec{k})+\frac{2 \pi}{k^{2}} \tan \alpha+2 \pi^{2} i \delta\left(k_{x}\right) \mathcal{P} \frac{1}{k_{y}}
\end{aligned}
$$

where $\alpha$ is the polar angel of the vector $\vec{k}$ and $\mathcal{P}$ means the principal-value distribution. Note that

$$
\begin{aligned}
\theta(\vec{k}) & =\theta_{0}(\vec{k})+2 \pi z \delta(\vec{k}), \\
\theta(-\vec{k}) & =\theta_{0}(-\vec{k})+\Delta_{0}(\vec{k})-\Delta \delta(\vec{k}) .
\end{aligned}
$$

It is easy to see that $\theta(\vec{k})$ respects the consistency condition

$$
\theta(\vec{k})-\theta(-\vec{k})=(2 z+1) \pi \delta(\vec{k})
$$

which can be found by Fourier transforming relation (11) . As far as the resemblance between the Greenberg model [4] and our multivalued picture of anyons is concerned, the latter does not contain any conjecture regarding the form of the representation (21) . This representation follows directly from the gauge transformation (8) which is the cornerstone of the Chern-Simons formulation of the fractional statistics in $2+1$ dimension. On the other hand, Greenberg's approach is more general ,since it does not depend on the dimensionality of the underlying space. 
We point out that the q-commutator algebras both in position space, Eq. (10), and in momentum space, Eq. (17), are the same and can be written in the $\mathcal{R}$-matrix approach [7] .For example, in position space we have

$$
\begin{aligned}
\tilde{\Psi}\left(\vec{r}_{1}\right) \tilde{\Psi}\left(\vec{r}_{2}\right)-\int d^{2} x \int d^{2} y \mathcal{R}\left(\vec{r}_{1}, \vec{r}_{2}, \vec{x}, \vec{y}\right) \tilde{\Psi}(\vec{y}) \tilde{\Psi}(\vec{x}) & =0 \\
\tilde{\Psi}\left(\vec{r}_{1}\right) \tilde{\Psi}^{\dagger}\left(\vec{r}_{2}\right)-\int d^{2} x \int d^{2} y \mathcal{R}\left(\vec{y}, \vec{r}_{1}, \vec{r}_{2}, \vec{x}\right) \tilde{\Psi}^{\dagger}(\vec{y}) \tilde{\Psi}(\vec{x}) & =\delta\left(\vec{r}_{1}-\vec{r}_{2}\right) .
\end{aligned}
$$

The $\mathcal{R}$-matrix is given by

$$
\mathcal{R}\left(\vec{r}_{1}, \vec{r}_{2}, \vec{r}_{3}, \vec{r}_{4}\right)=e^{-i \lambda \Delta} \delta\left(\vec{r}_{1}-\vec{r}_{3}\right) \delta\left(\vec{r}_{2}-\vec{r}_{4}\right)
$$

with multivalued matrix elements. Note that all quantites in a multivalud picture have a common multivalued unimodular factor $e^{i 2 \pi \lambda z}, z \in Z$, which has the property of unit element.

In order that the above $\mathcal{R}$-matrix algebra be associative, the following conditions have to be satisfied:

(i) the Yang-Baxter equation

$$
\begin{aligned}
& \iiint d^{2} x d^{2} y d^{2} z \mathcal{R}\left(\vec{r}_{1}, \vec{r}_{2}, \vec{x}, \vec{y}\right) \mathcal{R}\left(\vec{y}, \vec{z}, \vec{r}_{3}, \vec{r}_{4}\right) \mathcal{R}\left(\vec{x}, \vec{r}_{5}, \vec{r}_{6}, \vec{z}\right) \\
& =\iiint d^{2} x d^{2} y d^{2} z \mathcal{R}\left(\vec{r}_{2}, \vec{r}_{5}, \vec{x}, \vec{y}\right) \mathcal{R}\left(\vec{z}, \vec{x}, \vec{r}_{6}, \vec{r}_{3}\right) \mathcal{R}\left(\vec{r}_{1}, \vec{y}, \vec{z}, \vec{r}_{4}\right)
\end{aligned}
$$

(ii) hermiticity

$$
e^{-i \lambda \Delta}=e^{i \lambda \Delta}
$$

(iii) the Hecke condition

$$
(\check{\mathcal{R}}-1)(\check{\mathcal{R}}+1)=0
$$

where

$$
\begin{aligned}
\check{\mathcal{R}} & =P \mathcal{R} \quad \text { and } \\
P\left(\vec{r}_{1}, \vec{r}_{2}, \vec{r}_{3}, \vec{r}_{4}\right) & ={ }_{7}\left(\vec{r}_{1}-\vec{r}_{4}\right) \delta\left(\vec{r}_{2}-\vec{r}_{3}\right) .
\end{aligned}
$$


It is straightforward to show that the $\mathcal{R}$-matrix satisfies all the above conditions.Hence this proves the braiding properties of anyonic operators.

Finally,let us briefly comment on the clustering decomposition properties of the vacuum matrix elements of products of free-anyon multivalued fields.Like quon theory,anyonic physics in the Chern-Simons description is basically a nonlocal theory. Nevertheless,clustering holds for our nonrelativistic anyon theory. This follows from a similar type of arguments as in Ref. 四 using the algebra (10) .The twopoint function has to be replaced by $\delta\left(\vec{r}-\vec{r}^{\prime}\right)$ and the quon parameter q identified by the multivalued parameter $e^{i \lambda \Delta}$.

The next step is to study the physics of anyons in the single-valued picture and,in particular,the effect of cut-dependent terms upon the established q-deformed algebra of anyonic operators (17) .Further study of this problem will be reported elsewhere. 


\section{Acknowledgment}

This work was supported by the Scientific Fund of the Republic of Croatia. 


\section{References}

[1] D. P. Arovas, R. Schrieffer, F. Wilczek, and A. Zee,Nucl. Phys.B 251, $117(1985)$

G. Semenoff,Phys. Rev. Lett.61, 517(1988).

[2] T. Matsuyama,Phys. Rev. Lett. 228, 99(1989);

G. Semenoff and P. Sodano,Nucl. Phys.B 328, 753(1989);

R. Jackiw,Ann. Phys. 201, 83(1990).

[3] E. G. Floratos and T. N. Tomaras,Phys. Lett.B 251, 163(1990).

[4] O. W. Greenberg,Phys. Rev. Lett. 64, 705(1990); Phys. Rev.D. 43, 4111(1991).

[5] R. Jackiw and S. Y. Pi,Phys. Rev.D 42, 3500(1990).

[6] S. Forte, Rev. Mod. Phys. 64, 193(1992).

[7] D.B.Fairlie and C.K.Zachos,Phys.Lett.256B, 43 (1991). 